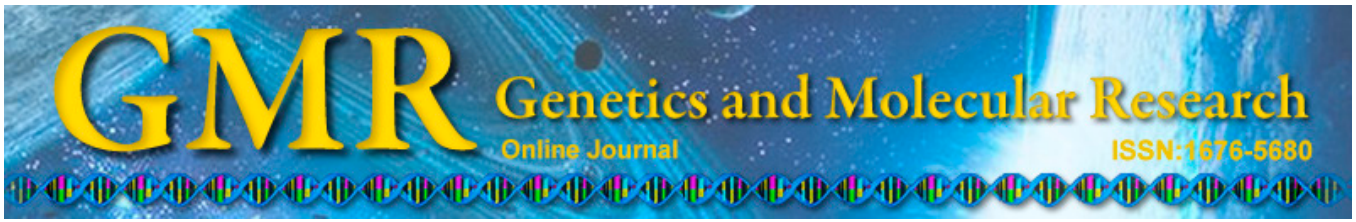

\title{
Branches of NF- $\kappa b$ signaling pathway regulate hepatocyte proliferation in rat liver regeneration
}

\author{
C.F. Chang ${ }^{1,2}$, W.M. Zhao ${ }^{1,2}$, J.X. Mei ${ }^{1,2}$, Y. Zhou ${ }^{1,2,3}$, C.Y. Pan ${ }^{1,2}$, T.T. Xu ${ }^{1,2}$ \\ and C.S. $\mathrm{Xu}^{1,2}$ \\ ${ }^{1}$ College of Life Science, Henan Normal University, Xinxiang, China \\ ${ }^{2}$ State Key Laboratory Cultivation Base for Cell Differentiation Regulation, \\ Henan Normal University, Xinxiang, China \\ ${ }^{3}$ College of Computer and Information Engineering, Henan Normal University, \\ Xinxiang, Henan, China \\ Corresponding author: C.S. Xu \\ E-mail: cellkeylab@126.com
}

Genet. Mol. Res. 14 (3): 7643-7654 (2015)

Received November 12, 2014

Accepted May 8, 2015

Published July 13, 2015

DOI http://dx.doi.org/10.4238/2015.July.13.9

\begin{abstract}
Previous studies have demonstrated that the nuclear factor $\kappa \mathrm{B}(\mathrm{NF}-\kappa \mathrm{B})$ pathway is involved in promoting cell proliferation. To further explore the regulatory branches and their sequence in the $\mathrm{NF}-\kappa \mathrm{B}$ pathway in the promotion of hepatocyte proliferation at the transcriptional level during rat liver regeneration, Rat Genome 230 2.0 array was used to detect the expression changes of the isolated hepatocytes. We found that many genes involved in the NF- $\kappa \mathrm{B}$ pathway (including 73 known genes and 19 homologous genes) and cell proliferation (including 484 genes and 104 homologous genes) were associated with liver regeneration. Expression profile function $\left(E_{p}\right)$ was used to analyze the biological processes. It was revealed that the NF$\kappa \mathrm{B}$ pathway promoted hepatocyte proliferation through three branches. Several methods of integrated statistics were applied to extract and screen key genes in liver regeneration, and it indicated that eight genes
\end{abstract}


may play a vital role in rat liver regeneration. To confirm the above predicted results, Ccnd1, Jun and Myc were analyzed using qRT-PCR, and the results were generally consistent with that of microarray data. It is concluded that 3 branches and 8 key genes involved in the NF- $\kappa \mathrm{B}$ pathway regulate hepatocyte proliferation during rat liver regeneration.

Key words: Rat liver regeneration; NF-kB signaling pathway; Key genes; Hepatocyte proliferation; Gene expression profile

\section{INTRODUCTION}

The liver comprises hepatocytes, bile duct epithelial cells and many other types of cells. Hepatocytes are the major hepatic cell type which account for $70-80 \%$ of the hepatic mass and $65 \%$ of total hepatic cells (Li et al., 2014), and they have many physiological functions including storage, metabolism, bile secretion, oxidation protection, detoxification, and production of many growth factors and cytokines (Fausto, 1995). Normally, only less than $0.01 \%$ hepatocytes of adult rat liver undergo mitosis (Xu et al., 2004). But a large number of quiescent hepatocytes can enter the cell cycle and proliferate rapidly to compensate for lost liver tissues after liver injury or partial hepatectomy $(\mathrm{PH})$. The process is called liver regeneration (Xu et al., 2012). It is well known that the liver has an outstanding capacity for regeneration, and this process involves various physiological and biochemical activities such as cell activation, de-differentiation, proliferation and its regulation, re-differentiation, and rebuilding of structure and function (Dabeva et al, 1998), where it is regulated by many signaling pathways (Brenner, 1998).

Previous studies have indicated that signal molecules such as tumor necrosis factor alpha (TNF- $\alpha$ ), growth factors, and chemotactic factors can promote cell proliferation through the NF- $\mathrm{KB}$ signaling pathway (Bassères and Baldwin, 2006). Among them, the TNF- $\alpha$ branch promotes cell proliferation through TNF- $\alpha \rightarrow$ TNFR $1 \rightarrow$ TRADD $\rightarrow$ TRAF $6 \rightarrow$ $\mathrm{IKK} \rightarrow \mathrm{IKB} \rightarrow \mathrm{NF}-\mathrm{kB}$ (Jackson-Bernitsas et al., 2007), the growth factor branch through growth factors $\rightarrow \mathrm{RTK} \rightarrow \mathrm{Ras} \rightarrow \mathrm{PI} 3 \mathrm{~K} \rightarrow \mathrm{Akt} \rightarrow \mathrm{NF}-\mathrm{kB}$ (Gui et al., 2011), and the chemotactic factor branch through chemokine $\rightarrow \mathrm{CR} \rightarrow \mathrm{G \alpha i} \rightarrow \mathrm{PI} 3 \mathrm{~K} \rightarrow \mathrm{Akt} \rightarrow \mathrm{NF}-\kappa \mathrm{B}$ (Van Sweringen et al., 2011). Generally, the NF- $\mathrm{BB}$ signaling pathway includes 238 genes, and cell proliferation includes 1784 genes, among which 136 genes are regulated by the NF- $\mathrm{kB}$ signaling pathway. Because many genes are involved in the NF- $\mathrm{\kappa B}$ signaling pathway and cell proliferation and because there are complex interactions between the genes, systems biology methods need to be applied to analyze the regulatory networks and the sequence of the NF- $\mathrm{KB}$ signaling pathway in modulating hepatocyte proliferation. The NF- $\mathrm{kB}$ signaling pathway includes many branches. Little is known as to which branches regulate the proliferation of hepatocytes during liver regeneration. In this study, we used Rat Genome 2302.0 array to determine the gene expression profiles of isolated hepatocytes from 10 time points of rat liver regeneration. The synergy between the genes involved in NF- $\kappa \mathrm{B}$ signal pathways and cell proliferation were calculated with a mathematical model of expression profile function $\left(E_{p}\right)$. The key genes in $\mathrm{NF}-\kappa \mathrm{B}$ signaling pathways and cell proliferation during rat liver regeneration were extracted and screened using an integrated statistics method and the way and mechanism of the key genes were analyzed using Pathway Studio 8.0. 
NF-kb pathway regulates hepatocyte proliferation

\section{MATERIAL AND METHODS}

\section{Isolation and identification of hepatocytes from rat regenerating liver}

The experimental procedure has been described in our previous studies. Briefly, rats of partial hepatectomy $\{\mathrm{PH}$ groups and operation control [also called sham operation (SO)] groups were prepared, and the isolated hepatocytes were identified by immunocytochemical analysis of two marker proteins, ALB and G6P (Wang et al., 2008). The purity of hepatocytes was $\geq 95 \%$. All experiments were carried out in accordance with the current Animal Protection Law of China.

\section{Rat Genome 2302.0 microarray detection and data analysis}

Total RNA was extracted and purified following the protocols previously described (Xu et al., 2010). Briefly, biotin-labeled cRNA was obtained using GeneChip In Vitro Transcript Labeling kit (ENZO Biochemical, New York, NY, USA), and then digested into 35- to 200-bp cRNA fragments. The hybridized arrays were washed and stained in GeneChip fluidics station 450 (Affymetrix Inc., Santa Clara, CA, USA). The arrays were then scanned and imaged with a GeneChip scanner 3000 (Affymetrix Inc.). The images reflecting gene expression abundance were converted into signal values and detection $P$ values using the Affymetrix GCOS 2.0 software. $\mathrm{P}<0.05$ indicated that the gene was present $(\mathrm{P}), \mathrm{P}<0.065$ meant marginal $(\mathrm{M})$, and when $\mathrm{P}>0.065$ meant totally absent (A). The signal values were normalized and used to calculate the relative value of every gene (ratio value), and their log ratio values were then calculated. To minimize the technical errors from microarray experiments, the average value of three independent detections by Rat Genome 2302.0 array was used for further analysis. Statistical and cluster analyses were conducted with these values using GeneMath, GeneSpring, Microsoft Excel and Pathway Studio 8.0 (Amon et al., 2003; Nikitin et al., 2003; Mulrane et al., 2008).

\section{qRT-PCR}

Total RNA was extracted and purified following the protocols previously reported (Wang and $\mathrm{Xu}, 2010$ ). Briefly, total RNA was reverse-transcribed using random primers and reverse transcription kit (Promega Corporation). The primers of Ccndl (NM_171992), Myc (NM_012603), Jun (NM_021835) and one internal control $\beta$-actin (NM_031144) were designed by the Primer Express 2.0 software. First-strand cDNA samples were subjected to quantitative PCR amplification by using SYBR ${ }^{\circledR}$ Green I on the Rotor-Gene 3000A (Corbett Robotics, Brisbane, Australia), and the copy numbers of target genes in each milliliter of the sample were calculated according to their corresponding standard curves. The quantitative PCR cycling conditions were $2 \mathrm{~min}$ at $95^{\circ} \mathrm{C}$, followed by 40 cycles for $15 \mathrm{~s}$ at $95^{\circ} \mathrm{C}, 15 \mathrm{~s}$ at $60^{\circ} \mathrm{C}$, and $30 \mathrm{~s}$ at $72^{\circ} \mathrm{C}$, and every sample was analyzed in triplicate. The controls included no template control (NTC), in which water replaced template, and no reverse transcription control (NRTC), in which reverse transcription enzyme AMV was omitted in the RT steps.

\section{Identification of NF- $\mathrm{B}$ signaling pathway- and cell proliferation-related genes}

First, the nomenclatures of "NF-kB signaling pathway" and "cell proliferation" were 
input into NCBI (www.ncbi.nlm.nih.gov) and RGD (rgd.mcw.edu) to collect rat, mouse and human genes associated with the NF- $\mathrm{kB}$ signaling pathway or cell proliferation. They were then collated and collected according to physiological pathway maps embodied by GENMAPP (www. genmapp.org), KEGG (www.genome.jp/kegg/pathway.html), and QIAGEN (www.qiagen.com/ geneglobe/pathways.aspx) (Salomonis et al., 2007; Antonov et al., 2010). Moreover, NF-кB signaling pathway-related genes, such as $N f k b, N f k b 2$, Rela, Relb, Rel(c-Rel), were input into TRED (http://rulai.cshl.edu/cgi-bin/TRED/tred.cgi?process=searchTFGeneForm), lymph TFDB (http:// www.iupui.edu/ tfinterx/activity.php) and into NF-kB (http://www.bu.edu/nf-kb/gene-resources/ target-genes/) to find out their downstream target genes in rat (Childress et al., 2007; Jiang et al., 2007), mouse and human, among which cell proliferation-related genes were identified with the NCBI database. In the end, the genes were reconfirmed through literature searches of pertinent articles. BLAST program was used to find out the homologous liver regeneration-related genes (Mojtaba et al., 2005), and the unknown genes homologous to NF-kB signaling pathway and/or cell proliferation-related genes were considered to possess the same function.

\section{Identification of significantly changed genes and liver regeneration-related genes during rat liver regeneration}

The log ratio values of $\mathrm{PH}$ normalized to that of control were used to calculate the relative value of every gene (ratio values), and the gene was considered significantly changed when its ratio values were $\geq 3$ or $\leq 0.33$ and considered non-significantly changed gene when its ratio values were between 2.99 and 0.34 during liver regeneration (Vardhanabhuti et al., 2006). At the same time, a $t$-test was used to analyze the significance of gene expression difference between PH and SO groups (de Menezes et al., 2004). The genes that were significantly changed in at least one time point during liver regeneration with significant difference $(0.01 \leq$ $\mathrm{P}<0.05)$ or extremely significant difference $(\mathrm{P} \leq 0.01)$ between $\mathrm{PH}$ and $\mathrm{SO}$, were referred to as associated with liver regeneration.

\section{Analysis of gene synergy}

The model $E_{p}$ was established by Xu and Jiang (2011), and used to analyze the synergy between NF- $\mathrm{KB}$ signaling pathway- and cell proliferation-related genes. In brief, Rat Genome 2302.0 could detect the expression changes of 20,489 genes of rat hepatocytes (Fukuhara et al., 2003), and the gene signal value in $\mathrm{PH}$ to that in SO groups was considered the ratio value for every gene. NF- $\mathrm{kB}$ signaling pathway- and hepatocyte proliferation-related genes were identified and collected from NCBI, RGD, GENMAPP, KEGG, and QIAGEN websites, and the $\log$ ratio values for these genes were calculated. In the end, the log ratio values for these genes were input into the $E_{p}$ formula to determine gene synergy.

\section{Exploring and screening of key genes during rat liver regeneration}

The key genes in rat liver regeneration were explored and screened according to the methods of integrated statistics established by Liu et al. (2013). In brief, Rat Genome 2302.0 could detect the expression changes of 20,489 genes of rat hepatocytes, and the ratio value of every gene were input into every formula to calculate gene expression difference in hepatocytes of rat liver regeneration. The top 1000 genes with maximal expression difference were 
then integrated for every formula. The top 1000 genes with highest frequency of occurrence from all algorithms were obtained. Only the genes belonging to the 1000 genes involved in the NF- $\mathrm{kB}$ signaling pathway and hepatocyte proliferation were considered as the key genes of hepatocytes in rat liver regeneration.

\section{Analysis of interactions among key genes and other genes}

Pathway Studio 8.0 was used to analyze and establish the interaction network for the whole genomic genes and then for the key genes of rat hepatocytes (Nikitin et al., 2003). Afterwards, the key genes were input into the interaction network of the whole genomic genes to identify their location, nodes, relationships and effects. Finally, the above information was used to enrich and rebuild the interaction network of the NF- $\mathrm{\kappa B}$ signal pathway- and hepatocyte proliferation-related genes, and then to identify the functions and pathways of the key genes.

\section{RESULTS}

\section{Expression change of NF- $\mathrm{KB}$ signaling pathway genes related to hepatocyte proliferation}

The data from NCBI, RGD, etc. and biological pathway maps in KEGG and QIAGEN etc. showed that 238 genes were involved in the NF- $\mathrm{KB}$ signaling pathway, among which 168 known genes and 20 unknown homologous genes were contained in the Rat Genome 230 2.0 array. The comparable analysis of $\mathrm{PH}$ and SO groups by a $t$-test indicated that 92 genes were significantly changed in hepatocytes of regenerating liver and were identified as liver regeneration-related genes, among which 63 known genes including $\operatorname{Tnf}$ and 15 unknown homologous genes were upregulated, 9 known genes including $E g f$ and 2 unknown homologous genes were downregulated, 6 genes including Lifr, Bf563716 and Aw532142 were up-/ downregulated (Table S1).

The data from NCBI, RGD, etc. and biological pathway maps in KEGG and QIAGEN showed that 1784 genes were involved in cell proliferation, among which 1314 known genes and 108 unknown homologous genes were included in the Rat Genome 2302.0 array. The comparable analysis of $\mathrm{PH}$ and $\mathrm{SO}$ groups by a $t$-test indicated that 588 genes were significantly changed in hepatocytes of regenerating liver and were identified as liver regenerationrelated genes, among which 428 genes including $C c n d 1$ and 84 unknown homologous genes were upregulated, and 52 genes such as Duspl and 17 unknown homologous genes were downregulated, while 4 genes including DSN1 and 3 unknown homologous genes were up-/ downregulated (Table S2).

The data from TRED, lymph TF DB, etc. show that 903 genes are regulated by $\mathrm{NF}-\kappa \mathrm{B}$ signaling pathway. Among them, 469 genes are contained in Rat Genome 2302.0 Array, and only 125 known genes and 11 unknown homologous genes were cell proliferation-related target genes. The difference analysis of gene expression in $\mathrm{PH}$ and SO groups indicated that 70 genes were significantly changed in hepatocytes of regenerating liver and were considered as liver regeneration-related genes, among which 53 known genes including Ccnd 1 etc. and 10 unknown homologous genes were upregulated and 5 known genes such as Dusp 1 and 1 unknown homologous gene Bm390716 were downregulated, while $K d r$ was up-/downregulated (Table 1). 


\begin{tabular}{|c|c|c|c|c|c|c|c|c|c|c|}
\hline \multirow[t]{2}{*}{ Gene symbol } & \multicolumn{10}{|c|}{ Recovery time (h) after partial hepatectomy (PH) } \\
\hline & 0 & 2 & 6 & 12 & 24 & 30 & 36 & 72 & 120 & 168 \\
\hline Ptpn1 & 1.00 & 3.37 & 2.46 & 1.89 & 1.29 & 1.39 & 1.23 & 1.52 & 1.73 & 1.53 \\
\hline Cdkn1a & 1.00 & 3.40 & 2.62 & 1.58 & 1.76 & 2.96 & 1.53 & 0.70 & 1.18 & 1.04 \\
\hline Pim1 & 1.00 & 1.21 & 1.13 & 0.88 & 1.30 & 0.98 & 0.90 & 1.03 & 1.01 & 0.88 \\
\hline AI598401 & 1.00 & 4.26 & 1.55 & 0.58 & 1.10 & 1.21 & 1.12 & 1.46 & 2.04 & 1.36 \\
\hline Igfbp1 & 1.00 & 4.77 & 1.04 & 1.59 & 2.38 & 1.52 & 1.15 & 2.41 & 1.89 & 1.85 \\
\hline Gadd45a & 1.00 & 6.92 & 2.88 & 0.91 & 1.12 & 1.10 & 0.95 & 1.23 & 1.06 & 0.78 \\
\hline Map3k8 & 1.00 & 14.44 & 2.28 & 2.21 & 1.55 & 1.18 & 1.08 & 1.89 & 2.58 & 1.90 \\
\hline Prl & 1.00 & 0.99 & 4.50 & 0.82 & 2.46 & 2.40 & 0.98 & 1.05 & 0.80 & 2.48 \\
\hline Il11 & 1.00 & 1.08 & 2.12 & 4.32 & 0.91 & 1.03 & 0.83 & 1.47 & 0.92 & 1.05 \\
\hline Skp2 & 1.00 & 1.03 & 0.62 & 1.03 & 3.71 & 2.44 & 2.66 & 0.98 & 0.84 & 1.20 \\
\hline Fgf8 & 1.00 & 1.13 & 2.89 & 1.41 & 1.33 & 4.40 & 1.53 & 1.60 & 1.56 & 2.84 \\
\hline $\mathrm{Illb}$ & 1.00 & 1.32 & 1.67 & 0.98 & 2.30 & 1.71 & 2.04 & 3.28 & 2.31 & 2.78 \\
\hline Ets1 & 1.00 & 0.56 & 0.71 & 0.69 & 1.29 & 0.98 & 1.29 & 4.23 & 1.85 & 1.34 \\
\hline S100a6 & 1.00 & 1.18 & 1.59 & 1.16 & 2.21 & 1.51 & 2.07 & 7.68 & 1.27 & 0.93 \\
\hline Tert & 1.00 & 0.96 & 0.44 & 0.63 & 1.64 & 1.25 & 1.84 & 2.80 & 1.71 & 3.35 \\
\hline $\mathrm{Bcl} 3$ & 1.00 & 4.35 & 3.20 & 2.54 & 1.66 & 1.07 & 1.08 & 1.77 & 2.06 & 1.82 \\
\hline Bmp2 & 1.00 & 0.37 & 0.75 & 0.51 & 1.08 & 0.73 & 1.18 & 6.60 & 1.92 & 1.10 \\
\hline BG376853 & 1.00 & 2.18 & 6.94 & 3.18 & 4.81 & 1.01 & 1.18 & 5.90 & 2.98 & 2.85 \\
\hline Ccnd2 & 1.00 & 0.77 & 0.80 & 0.68 & 1.37 & 2.01 & 2.57 & 7.29 & 1.16 & 1.38 \\
\hline BG380633 & 1.00 & 1.00 & 1.27 & 0.91 & 2.56 & 3.67 & 4.50 & 10.33 & 2.60 & 2.53 \\
\hline Hmox 1 & 1.00 & 13.12 & 7.99 & 8.41 & 2.04 & 2.96 & 3.54 & 4.47 & 6.75 & 2.87 \\
\hline BF282029 & 1.00 & 1.45 & 2.40 & 5.59 & 3.68 & 4.12 & 3.21 & 3.42 & 2.67 & 1.98 \\
\hline Jun & 1.00 & 3.29 & 2.68 & 1.97 & 3.97 & 1.54 & 1.91 & 2.14 & 1.75 & 2.18 \\
\hline Myc & 1.00 & 5.43 & 1.77 & 1.84 & 5.10 & 1.75 & 1.14 & 1.48 & 1.27 & 1.33 \\
\hline Pcna & 1.00 & 1.19 & 1.02 & 1.25 & 3.50 & 3.83 & 2.77 & 0.81 & 0.80 & 0.82 \\
\hline Tgfb1 & 1.00 & 1.19 & 1.14 & 1.44 & 1.88 & 1.60 & 1.74 & 7.05 & 5.16 & 2.01 \\
\hline $\mathrm{Ngf}$ & 1.00 & 4.81 & 1.11 & 2.62 & 0.99 & 1.26 & 2.17 & 2.38 & 3.42 & 4.68 \\
\hline Wt1 & 1.00 & 2.55 & 3.19 & 3.63 & 2.04 & 2.48 & 1.65 & 3.60 & 1.96 & 1.78 \\
\hline Ptprv & 1.00 & 1.72 & 4.37 & 2.56 & 1.20 & 5.91 & 1.75 & 4.59 & 1.18 & 2.59 \\
\hline Tnf & 1.00 & 1.71 & 4.98 & 2.26 & 1.96 & 2.80 & 3.65 & 3.40 & 2.03 & 1.39 \\
\hline Creb1 & 1.00 & 1.76 & 6.02 & 1.94 & 1.55 & 2.42 & 4.55 & 3.29 & 1.15 & 2.47 \\
\hline Birc5 & 1.00 & 1.14 & 0.69 & 0.77 & 3.63 & 5.08 & 5.44 & 2.27 & 1.15 & 0.74 \\
\hline Brca1 & 1.00 & 1.17 & 1.19 & 1.06 & 5.29 & 4.42 & 4.00 & 1.65 & 1.27 & 1.30 \\
\hline BF284903 & 1.00 & 0.83 & 1.01 & 0.62 & 8.73 & 4.72 & 6.56 & 2.90 & 1.86 & 1.22 \\
\hline Ccr5 & 1.00 & 1.57 & 2.36 & 1.27 & 3.00 & 7.14 & 1.41 & 3.53 & 1.45 & 2.95 \\
\hline Il2ra & 1.00 & 1.34 & 1.57 & 1.79 & 1.34 & 3.32 & 3.84 & 2.62 & 1.17 & 3.29 \\
\hline $\mathrm{Cd} 74$ & 1.00 & 0.73 & 1.57 & 0.77 & 1.82 & 1.30 & 1.27 & 7.17 & 3.08 & 3.39 \\
\hline Hgf & 1.00 & 1.37 & 3.23 & 2.73 & 6.76 & 3.91 & 2.54 & 18.65 & 1.43 & 2.77 \\
\hline Cond1 & 1.00 & 1.75 & 1.24 & 3.28 & 4.14 & 8.92 & 6.57 & 2.11 & 2.61 & 1.40 \\
\hline Il10 & 1.00 & 1.02 & 0.83 & 0.89 & 5.76 & 5.39 & 4.45 & 3.93 & 1.91 & 0.95 \\
\hline Brca2 & 1.00 & 1.80 & 0.49 & 1.79 & 11.70 & 11.30 & 10.51 & 7.80 & 2.09 & 1.23 \\
\hline Pla2g2a & 1.00 & 0.68 & 1.09 & 0.70 & 21.42 & 1.73 & 4.59 & 151.14 & 4.10 & 1.13 \\
\hline Fyn & 1.00 & 0.61 & 2.53 & 1.47 & 2.95 & 2.63 & 3.16 & 9.87 & 4.19 & 3.27 \\
\hline Junb & 1.00 & 8.32 & 4.32 & 3.13 & 3.05 & 1.05 & 0.85 & 2.15 & 3.31 & 1.92 \\
\hline Lipg & 1.00 & 8.83 & 1.59 & 1.20 & 10.64 & 8.62 & 12.71 & 1.60 & 2.70 & 7.77 \\
\hline Fos & 1.00 & 5.02 & 1.12 & 4.73 & 14.96 & 2.31 & 1.04 & 5.21 & 2.76 & 4.23 \\
\hline Pten & 1.00 & 0.97 & 0.70 & 0.93 & 0.77 & 1.20 & 0.83 & 0.94 & 1.09 & 1.20 \\
\hline BE103748 & 1.00 & 1.43 & 0.57 & 2.59 & 5.95 & 3.58 & 4.86 & 8.84 & 3.07 & 2.80 \\
\hline Tnfrsf9 & 1.00 & 3.80 & 1.53 & 9.55 & 3.36 & 2.51 & 3.35 & 4.25 & 3.70 & 2.42 \\
\hline Ppara & 1.00 & 7.14 & 0.68 & 0.87 & 2.81 & 6.00 & 6.63 & 3.78 & 3.35 & 5.07 \\
\hline BM390716 & 1.00 & 1.23 & 0.25 & 0.24 & 0.47 & 0.89 & 0.69 & 1.53 & 1.72 & 2.00 \\
\hline Serpine1 & 1.00 & 16.12 & 3.33 & 22.52 & 4.81 & 4.34 & 2.76 & 7.52 & 1.83 & 1.63 \\
\hline Timp1 & 1.00 & 1.88 & 4.32 & 4.90 & 14.94 & 6.13 & 8.45 & 33.89 & 2.66 & 1.33 \\
\hline Myod1 & 1.00 & 1.90 & 15.60 & 11.59 & 10.62 & 2.51 & 1.92 & 15.49 & 3.10 & 11.00 \\
\hline S100a10 & 1.00 & 2.11 & 2.08 & 6.19 & 3.14 & 9.83 & 5.40 & 7.65 & 3.60 & 2.87 \\
\hline Cebpd & 1.00 & 14.03 & 5.71 & 7.62 & 3.23 & 2.16 & 2.24 & 5.84 & 6.59 & 5.29 \\
\hline Cxcl1 & 1.00 & 47.47 & 28.53 & 15.40 & 6.92 & 0.59 & 2.58 & 6.94 & 13.60 & 4.33 \\
\hline $\mathrm{S} 100 \mathrm{a} 4$ & 1.00 & 1.13 & 3.98 & 3.49 & 13.84 & 12.12 & 17.77 & 24.62 & 6.43 & 1.30 \\
\hline Rela & 1.00 & 1.38 & 1.21 & 1.28 & 1.14 & 1.05 & 1.00 & 0.95 & 1.11 & 1.02 \\
\hline
\end{tabular}




\begin{tabular}{|c|c|c|c|c|c|c|c|c|c|c|}
\hline \multirow[t]{2}{*}{ Gene symbol } & \multicolumn{10}{|c|}{ Recovery time (h) after partial hepatectomy (PH) } \\
\hline & 0 & 2 & 6 & 12 & 24 & 30 & 36 & 72 & 120 & 168 \\
\hline BF419700 & 1.00 & 1.94 & 1.34 & 5.47 & 6.20 & 3.70 & 7.14 & 5.62 & 3.69 & 9.35 \\
\hline Cyld & 1.00 & 0.93 & 1.00 & 1.01 & 1.00 & 1.08 & 0.95 & 1.10 & 1.08 & 1.04 \\
\hline BF284574 & 1.00 & 2.53 & 3.94 & 4.47 & 5.27 & 5.07 & 4.62 & 6.51 & 3.21 & 5.13 \\
\hline $\mathrm{Bcl} 2$ & 1.00 & 3.07 & 1.85 & 4.39 & 3.60 & 5.06 & 4.98 & 6.04 & 3.06 & 3.15 \\
\hline BE109363 & 1.00 & 1.72 & 1.79 & 3.19 & 2.66 & 2.07 & 2.98 & 3.30 & 3.46 & 3.58 \\
\hline BF398185 & 1.00 & 13.63 & 22.68 & 2.63 & 17.98 & 16.62 & 30.78 & 2.94 & 22.32 & 13.56 \\
\hline Csf3 & 1.00 & 3.07 & 4.05 & 5.68 & 1.46 & 5.89 & 3.47 & 4.21 & 3.66 & 3.81 \\
\hline Gadd $45 b$ & 1.00 & 148.86 & 34.04 & 17.96 & 21.46 & 29.10 & 10.87 & 42.88 & 25.52 & 11.86 \\
\hline Ppard & 1.00 & 0.70 & 0.24 & 0.63 & 0.49 & 0.35 & 1.11 & 0.78 & 0.48 & 1.11 \\
\hline Tbxa2r & 1.00 & 1.41 & 1.04 & 0.64 & 0.89 & 0.64 & 0.25 & 1.16 & 0.77 & 0.93 \\
\hline I15 & 1.00 & 1.19 & 1.33 & 0.48 & 1.29 & 0.69 & 0.32 & 0.96 & 1.29 & 0.79 \\
\hline Met & 1.00 & 1.43 & 0.36 & 0.58 & 0.64 & 0.75 & 0.51 & 0.23 & 0.36 & 0.70 \\
\hline Dusp1 & 1.00 & 0.33 & 0.50 & 0.23 & 0.72 & 0.36 & 0.19 & 0.47 & 0.53 & 0.96 \\
\hline $\mathrm{Kdr}$ & 1.00 & 0.22 & 0.33 & 0.12 & 1.18 & 0.77 & 1.16 & 5.35 & 2.33 & 1.57 \\
\hline
\end{tabular}

*Values in bold represent the expression abundance of upregulated genes, those in light gray that of the downregulated. The symbols in black ground indicate the unknown genes homologous to the above known genes.

The expression trends of 4 target genes including $C c n d 1, M Y C$ and $J U N$ were detected by real-time RT-PCR and were compared to the results by gene chip detection, which showed that their expression trends detected by the above two methods were generally consistent (Figure 1). Other genes detected by real-time RT-PCR in our previous papers also gave the same results (Xu and Zhang, 2009), indicating that the array results were reliable for further analysis.

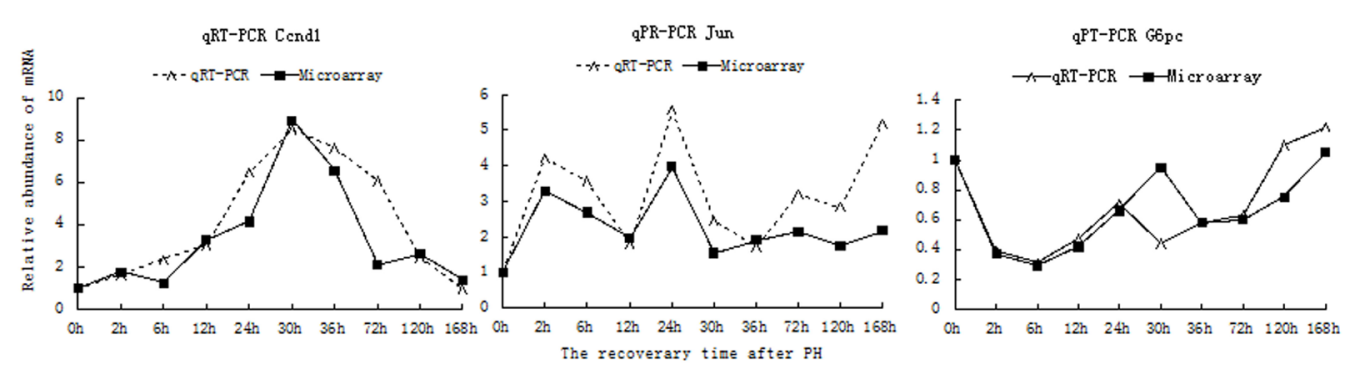

Figure 1. mRNA expression of four selected genes measured by microarrays and RT-PCR. Solid line presented the results of RT-PCR and dotted line that of Rat Genome 2302.0 Array.

\section{Relationships between the signal transduction action of three branches of NF-кB signaling pathway and hepatocyte proliferation}

$E_{p}$ was used to analyze the synergy between NF-kB signaling pathway-related genes in hepatocytes, and it demonstrated that the signal transduction activity of NF- $\mathrm{kB}$ signaling pathway was increased during liver regeneration. $E_{P}$ values of genes related to three main branches of the NF- $\mathrm{kB}$ signaling pathway were also higher than those in normal control and $\mathrm{SO}$ groups (Figure 2). 


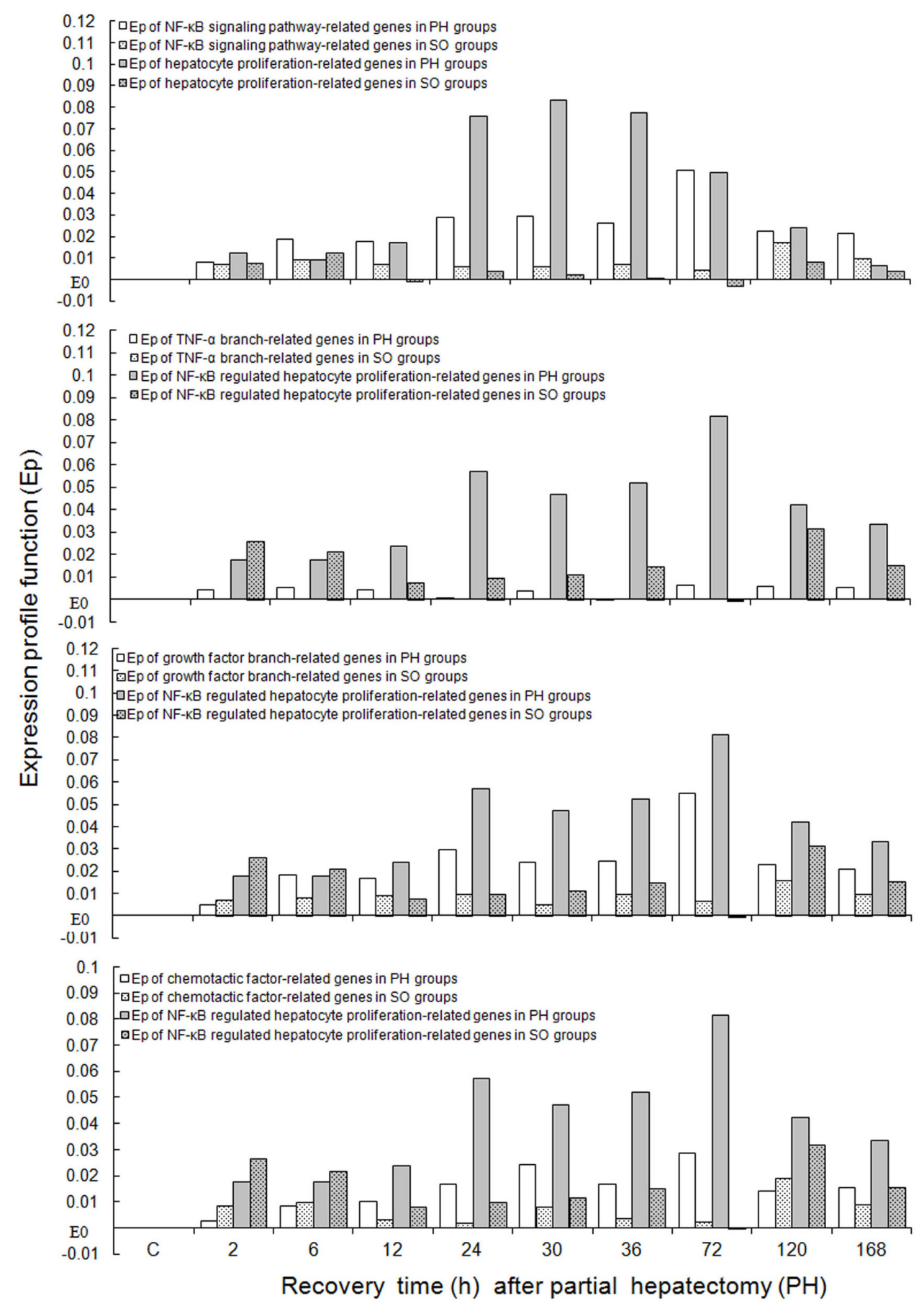

Figure 2. Relationships between the roles of NF- $\mathrm{kB}$ signaling pathway branches and that of their regulated hepatocyte proliferation. 
$E_{p}$ values of hepatocyte proliferation-related genes were greater than those in normal control and SO groups, and peaked at $30 \mathrm{~h}$ after PH. Furthermore, $E_{p}$ values of TNF- $\alpha$, growth factor and chemotactic factor branch-regulated hepatocyte proliferation-related genes were also higher than those in normal control and SO groups, and were all significantly higher than those at $72 \mathrm{~h}$ (Figure 2).

The relationships between the signal transduction activity of NF-kB signaling pathway and cell proliferation were analyzed by $E_{p}$, and $E_{p}$ values of NF-kB signaling pathway-hepatocyte proliferation-related genes were higher than those in normal control and $\mathrm{SO}$ groups.

In detail, $E_{P}$ of TNF- $\alpha$ branch-related genes was higher than those in normal control and $\mathrm{SO}$ groups since all the time after $\mathrm{PH}$, and $E_{P}$ of its regulatory hepatocyte proliferationrelated genes was higher since $12 \mathrm{~h} . E_{P}$ of growth factor branch-related genes was higher than those in normal control and $\mathrm{SO}$ groups except at $2 \mathrm{~h}$, while its regulatory hepatocyte proliferation-related genes was higher since $12 \mathrm{~h} . E_{P}$ of chemotactic factor branch-related genes were higher than those in normal control and SO groups except at 2, 6, and $120 \mathrm{~h}$, and its regulatory hepatocyte proliferation-related genes were higher since $12 \mathrm{~h}$.

\section{Key genes in both NF-кB signaling pathway and hepatocyte proliferation, and their functions during rat liver regeneration}

Several methods of integrated statistics were applied to analyze the difference in gene expression between $\mathrm{PH}$ and SO groups, and they indicated that the differences in eight genes including Tnfrsf12a, Cxcl12, Pik3r1, Lifr, Ccnd1, Dusp1, Myc, and Timp1 were maximal, demonstrating that the eight genes may play important roles in rat liver regeneration. TNF- $\alpha$ and its receptor TNFRSF12A together activate the NF-kB/TNFa branch, and they were both upregulated. Growth factor combined with its receptor gene Lifr activates Pik3r1, while Cxcl12 can also activate Pik3rl through the NF-kB/chemotactic factor branch. Among the above genes, Cxcll2 was downregulated, Pik3rl upregulated, and Lifr up-/downregulated. Bf419700, homologous to one member of the NF-kB family, Rela, was upregulated by the above three branches, and then Bf419700 promoted the expression of three cell proliferationstimulatory genes, Ccnd1, Myc and Timpl and suppressed the expression of one cell proliferation-inhibitory gene Duspl (Figure 3).

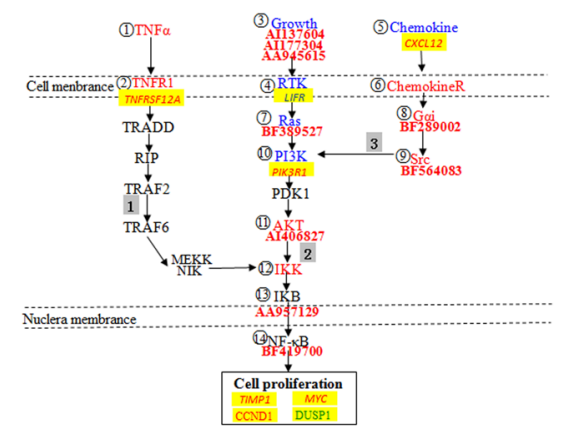

Figure 3. Regulatory effect of the three branches of NF- $\mathrm{kB}$ signaling pathway on hepatocyte proliferation. Symbols in red represent meaningful upregulated genes, green means downregulated, blue denotes up/downregulated and black is insignificantly changed. Symbols in red bold indicate the genes homologous to the upper gene. The symbols in black bold shows the activity studied in this paper. Symbols with yellow ground represent the key genes. Numbers in circle are consistent with the ordinal numbers of proteins in Table S1. 


\section{DISCUSSION}

In this study, the expression profile of TNF- $\alpha$ branch-related genes was detected using Rat Genome 2302.0 array at the transcriptional level during rat liver regeneration. It was found that $T N F-\alpha$ was upregulated at 36-72 $\mathrm{h}$ and its receptor Tnfrsf $12 a$ upregulated at 2-36 $\mathrm{h}$, and that $B f 419700$ (homologue of transcription factor NF-kB family member Rela) showed increased mRNA levels at 12-168 h. Key gene $C c n d 1$ was found upregulated at 12-36 h during rat liver regeneration, Timp1 showed enhanced expression at $72 \mathrm{~h}$, while Duspl was downregulated at 2, 12, and $36 \mathrm{~h}$ (Table S1). Systems biology methods were applied to analyze the physiological processes indicated by gene expression changes, and it revealed that the TNF- $\alpha$ branch and its regulation of hepatocyte proliferation activity were enhanced during rat liver regeneration (Figure 1). The results above were consistent with those of Jackson-Bernitsas et al. (2007) and Gallucci et al. (2000), who found a correlation between the signal transduction activity of the TNF- $\alpha$ branch of the NF- $\mathrm{kB}$ signaling pathway and hepatocyte proliferation activity. On the basis of the above results, the possible relationship between the TNF- $\alpha$ branch and hepatocyte proliferation was deduced as follows. The overexpression of TNF- $\alpha$ and its receptor Tnfrsf $12 a$ activates transcription factor NF- $\mathrm{kB}$ family member Rela's homologous gene $A A 998997$, which augments hepatocyte proliferation activity by upregulating the expression of the cell proliferation-promoting genes $C c n d 1, M y c$, and Timp1 and downregulating the expression of the cell proliferation-inhibiting gene Dusp1, resulting in enhanced hepatocyte proliferation.

The expression of growth factor branch-related genes showed that hepatocyte growth factor (HGF) was upregulated at 6, 24-30, and $72 \mathrm{~h}$ in rat liver regeneration, epidermal growth factor (EGF) at 12 and $30 \mathrm{~h}$, and nerve growth factor (NGF) at 2 and 120-168 h. Several members of fibroblast growth factor (FGF) family were upregulated during liver regeneration, with $F g f 1$ at 30 h, Fgf4 at 72 h, Fgf5 at 2, $12-24$ and 36 h, Fgf7 at 6 h, Fgf8 at 30 h, Fgfl2 at 6, 24-73 and $168 \mathrm{~h}, \mathrm{Fgfl} 3$ at 24-30 and 72-168 h, Fgfl during the whole process of liver regeneration, and $F g f 21$ at $12 \mathrm{~h}$. Rtk/Lifr, the growth factor receptor, was increased at $2 \mathrm{~h}$. Rtk-activated Bf389527 (the homologous gene of Kras) was elevated at the mRNA level at $6 \mathrm{~h}$ (Table S1). The activities of physiological processes reflected by the expression changes were analyzed then by systems biology methods, and both growth factor branches and its regulation of hepatocyte proliferation activity were elevated in rat liver regeneration (Figure 1). The above results were consistent with those of other authors (Gezginci-Oktayoglu et al., 2010; Paranjpe et al., 2010; Gui et al., 2011; Tsai and Wang, 2011; Wu et al., 2011), who demonstrated the correlation between the signal transduction activity of the growth factor branch of the NF- $\kappa \mathrm{B}$ signaling pathway and hepatocyte proliferation activity. The above results helped deduce the following possible connection between the growth factor branch and hepatocyte proliferation activity. Upregulation of the above four kinds of growth factors and their receptor gene Lifr promotes the overexpression of the Kras homologous gene Bf389527, which activates $B f 419700$ (the homologous gene of transcription factor NF- $\kappa B$ family member Rela), $B f 419700$ then enhances hepatocyte proliferation by upregulating cell proliferation-promoting genes Ccnd1, Myc, and Timp1 and downregulating cell proliferation-inhibiting gene Duspl.

Microarray analysis of chemotactic factor branch-related genes pointed out that Cxcl12 was downregulated at 6-24 h, while its receptor-activated gene Gnail was upregulated at 6-30 and 72-168 h, and its homologous gene $B f 289002$ at 6,12, and $72 \mathrm{~h}$ during rat 
liver regeneration (Table S1). The activities of physiological processes reflected by expression changes were analyzed by systems biology methods, and both the chemotactic factor branches and its regulation of hepatocyte proliferation activity were found increased in rat liver regeneration (Figure 1). This result was consistent with the conclusion of Van Sweringen et al. (2011), who demonstrated the correlation between the signal transduction activity of the chemotactic factor branch of the NF- $\mathrm{KB}$ signaling pathway and hepatocyte proliferation activity (Clarke et al., 2011; Van Sweringen et al., 2011). The above results led to the speculation that the downregulation of $\mathrm{Cxcl12}$ and upregulation of its receptor-activated gene Gnail and its homologous genes $B f 289002$ together activate transcription factor NF- $\mathrm{kB}$ family member Rela's homologous gene $B f 419700$, which augment hepatocyte proliferation activity by upregulating the expression of cell proliferation-promoting genes Ccnd1, Myc, and Timpl and downregulating the expression of cell proliferation-inhibiting gene Dusp 1, ultimately enhancing hepatocyte proliferation.

This study confirmed that 3 branches and 8 key genes involved in the NF- $\kappa B$ signaling pathway regulate the proliferation of hepatocytes during rat liver regeneration. In the future, gene knockout, overexpression, RNA interference, and some other methods will be used to further study the mechanisms about how every branch of the NF- $\mathrm{BB}$ signaling pathway regulates hepatocyte proliferation in rat liver regeneration.

\section{ACKNOWLEDGMENTS}

Research supported by National Basic Research "973" Pre-Research Program of China (Grant \#2012CB722304), Natural Science Foundation of China (\#31201093), Natural Science Foundation of Henan (\#122300413207 and No. 132300413208), Major Scientific and Technological Projects of Henan (\#111100910600), and Doctorate Research Fund of Henan Normal University (\#11134).

\section{Supplementary material}

\section{REFERENCES}

Amon RR, DuBois DC, Pearson KE, Stephan DA, et al. (2003). Gene arrays and temporal patterns of drug response: corticosteroid effects on rat liver. Funct. Integr. Genomics 3: 171-179.

Antonov AV, Schmidt EE, Dietmann S, Krestyaninova M, et al. (2010). R spider: a network-based analysis of gene lists by combining signaling and metabolic pathways from Reactome and KEGG databases. Nucleic Acids Res. 38: 78-83.

Bassères DS and Baldwin AS (2006). Nuclear factor-kappaB and inhibitor of kappaB kinase pathways in oncogenic initiation and progression. Oncogene 25: 6817-6830.

Brenner DA (1998). Signal transduction during liver regeneration. J. Gastroenterol. Hepatol. 13 (Suppl): 93-95.

Childress PJ, Fletcher RL and Perumal NB (2007). LymphTF-DB: a database of transcription factors involved in lymphocyte development. Genes Immun. 8: 360-365.

Clarke C, Kuboki S, Sakai N, Kasten KR, et al. (2011). CXC chemokine recEptor-1 is expressed by hepatocytes and regulates liver recovery after hepatic ischemia/reperfusion injury. Hepatology 53: 261-271.

Dabeva MD, Laconi E, Oren R, Petkov PM, et al. (1998). Liver regeneration and alpha-fetoprotein messenger RNA expression in the retrorsone model for hepatocyte transplantation. Cancer Res. 58: 5825-5834.

de Menezes RX, Boer JM and van Houwelingen HC (2004). Microarray data analysis: a hierarchical T-test to handle heteroscedasticity. Appl. Bioinformatics 3: 229-235.

Fausto N, Laird AD and Webber EM (1995). Liver regeneration. 2. Role of growth factors and cytokines in hepatic regeneration. FASEB J. 9: 1527-1536.

Fukuhara Y, Hirasawa A, Li XK, Kawasaki M, et al. (2003). Gene expression profile in the regenerating rat liver after 
partial hepatectomy. J. Hepatol. 38: 784-792.

Gallucci RM, Simeonova PP, Toriumi W and Luster MI (2000). TNF-alpha regulates transforming growth factor-alpha expression in regenerating murine liver and isolated hepatocytes. J. Immunol. 164: 872-878.

Gezginci-Oktayoglu S, Sacan O, Yanardag R, Karatug A, et al. (2010). Exendin-4 improves hepatocyte injury by decreasing proliferation through blocking NGF/TrkA in diabetic mice. Peptides 32: 223-231.

Gui Y, Yeganeh M, Ramanathan S, Leblanc C, et al. (2011). SOCS1 controls liver regeneration by regulating HGF signaling in hepatocytes. J. Hepatol. 55: 1300-1308.

Jackson-Bernitsas DG, Ichikawa H, Takada Y, Myers JN, et al. (2007). Evidence that TNF-TNFR1-TRADD-TRAF2RIP-TAK1-IKK pathway mediates constitutive NF-kappaB activation and proliferation in human head and neck squamous cell carcinoma. Oncogene 26: 1385-1397.

Jiang C, Xuan Z, Zhao F and Zhang MQ (2007). TRED: a transcriptional regulatory element database, new entries and other development. Nucleic Acids Res. 35: 137-140.

Li MH, Zhou XC, Mei JX, Geng XF, et al. (2014). Study on the activity of the signaling pathways regulating hepatocytes from G0 phase into G1 phase during rat liver regeneration. Cell Mol. Biol. Lett. 19: 181-200.

Liu YQ, Huang Y, Zhou Y, Li JT, et al. (2013). Predicting rat hepatocyte key genes in liver regeneration by same kind extraction method coupled with sequential-forward-selection method. Henan Sci. 7: 964-967.

Mojtaba Sa, Abdolreza V, Nazanin P and Mahmoud M (2005). Sequence homology: a poor predictive value for profilins cross-reactivity. Clin. Mol. Allergy 3: 13.

Mulrane L, Rexhepaj E, Smart V, Callanan JJ, et al. (2008). Creation of a digital slide and tissue microarray resource from a multi-institutional predictive toxicology study in the rat: an initial report from the PredTox group. Exp. Toxicol. Pathol. 60: 235-245.

Nikitin A, Egorov S, Daraselia N and Mazo I (2003). Pathway studio - the analysis and navigation of molecular networks. Bioinformatics 19: 2155-2157.

Paranjpe S, Bowen WC, Tseng GC, Luo JH, et al. (2010). RNA interference against hepatic epidermal growth factor receptor has suppressive effects on liver regeneration in rats. Am. J. Pathol. 176: 2669- 2681.

Salomonis N, Hanspers K, Zambon AC, Vranizan K, et al. (2007). GenMAPP 2: new features and resources for pathway analysis. BMC Bioinformatics 8: 217.

Tsai SM and Wang WP (2011). Expression and function of fibroblast growth factor (FGF) 7 during liver regeneration. Cell Physiol. Biochem. 27: 641-652.

Van Sweringen HL, Sakai N, Tevar AD, Burns JM, et al. (2011). CXC chemokine signaling in the liver: impact on repair and regeneration. Hepatology 54: 1445-1453.

Vardhanabhuti S, Blakemore SJ, Clark SM, Ghosh S, et al. (2006). A comparison of statistical tests for detecting differential expression using Affymetrix oligonucleotide microarrays. OMICS 10: 555-566.

Wang GP and Xu CS (2010). Reference gene selection for real-time RT-PCR in eight kinds of rat regenerating hepatic cells. Mol. Biotechnol. 46: 49-57.

Wang WB, Xie LF, Wang W, Wang L, et al. (2008). Isolation, purity and identification of hepatocytes in rat normal liver and regenerating liver. Henan Sci. 12: 1492-1498.

Wu AL, Coulter S, Liddle C, Wong A, et al. (2011). FGF19 regulates cell proliferation, glucose and bile acid metabolism via FGFR4-dependent and independent pathways. PLoS One 6: e17868.

Xu CS and Zhang JB (2009). Research on the Functional Genomics of the Rat Regenerating Liver. Higher Education Press, Beijing.

Xu CS and Jiang Y (2011). Expression profile function and its applications in microarray data analysis. Henan Science 15: 229-231.

Xu CS, Chang CF, Yuan JY, Han HP, et al. (2004). Identification and characterization of 177 unreported genes associated with liver regeneration. Genomics Proteomics Bioinformatics 2: 109-118.

Xu CS, Chen XG, Chang CF, Wang GP, et al. (2010). Transcriptome analysis of hepatocytes after partial hepatectomy in rats. Dev. Genes Evol. 220: 263-274.

Xu CS, Yang YJ, Yang JY, Chen XG, et al. (2012). Analysis of the role of the integrin signaling pathway in hepatocytes during rat liver regeneration. Cell Mol. Biol. Lett. 17: 274-288. 